\title{
Homomorphisms of modules associated with polynomial matrices with infinite elementary divisors
}

\author{
Pudji Astuti \\ Harald K. Wimmer \\ Departement of Mathematics \\ Mathematisches Institut \\ and Natural Sciences \\ Universität Würzburg \\ Institut Teknologi Bandung \\ Bandung 40132 \\ D-97074 Würzburg \\ Indonesia $^{1}$ \\ Germany
}

\footnotetext{
${ }^{1}$ The research of the first author was supported by Deutscher Akademischer Austauschdienst under Award No. A/98/25636.
} 


\begin{abstract}
If the inverse of a nonsingular polynomial matrix $L$ has a polynomial part then one can associate with $L$ a module over the ring of proper rational functions, which is related to the structure of L at infinity. In this paper we characterize homomorphisms of such modules.
\end{abstract}

Mathematical Subject Classifications (2010): 15B33, 13C12 93B25

Keywords: polynomial matrices, proper rational functions, module homomorphisms, duality, infinite elementary divisors, coprimeness 


\section{Introduction}

According to Rosenbrock [6] a transfer matrix $G \in K^{m \times p}$ of rational functions over a field $K$ admits a generalized state space realization

$$
G(s)=\left(\begin{array}{ll}
C_{1} & C_{2}
\end{array}\right)\left(\begin{array}{cc}
s I-A_{1} & 0 \\
0 & s N_{2}-I
\end{array}\right)\left(\begin{array}{l}
B_{1} \\
B_{2}
\end{array}\right)
$$

such that

$$
G_{1}(s)=C_{1}\left(s I-A_{1}\right)^{-1} B_{1}
$$

is the strictly proper part and

$$
G_{2}(s)=C_{2}\left(s N_{2}-I\right)^{-1} B_{2}
$$

where $N_{2}$ is nilpotent, is the polyonomial part of $G$. It is well known that the realizations (1.1) and (1.2) can be constructed by module theoretic approaches. In the case of (1.1) a construction is due to Fuhrmann [2]. For a realization theory of anticausal input output maps we refer to Conte and Perdon [1]. To describe the polynomial models that serve as state spaces for (1.1) and (1.2) we use the following notation. A rational function $f \in K(s)$ is called proper or causal (resp. strictly proper or strictly causal) if $f=0$ or if $f \neq 0$ and $f=p / q, p, q \in K[s], q \neq 0$, and $\operatorname{deg} p \leq \operatorname{deg} q$ (resp. $\operatorname{deg} p<\operatorname{deg} q)$. Let $K_{\infty}(s)$ denote the ring of proper rational functions over $K$. Then

$$
K(s)=K[s] \oplus s^{-1} K_{\infty}(s) .
$$

To (1.3) correspond projection operators

$$
\pi_{-}: K(s) \rightarrow s^{-1} K_{\infty}(s)
$$

and

$$
\pi_{+}=\left(I-\pi_{-}\right): K(s) \rightarrow K[s]
$$

Put

$$
(f)_{0}=\left(\pi_{+} f\right)(0), f \in K(s) .
$$

The decomposition (1.3), the projections $\pi_{-}$and $\pi_{+}$, and definition (1.4) extend naturally from $K(s)$ to $K^{n}(s)$ and $K^{m \times p}(s)$.

Let $G \in K^{m \times p}(s)$ have a realization

$$
G=W_{1}+P_{1} D_{1}^{-1} Q_{1}
$$

where $W_{1}, P_{1}, Q_{1}, D_{1}$ are polynomial matrices, with $D_{1}$ of size $n_{1} \times n_{1}$. In Fuhrmann's theory [4] a state space for a realization (1.1) of $\pi_{-} G$ is provided by

$$
V_{D_{1}}=K_{1}^{n}[s] / D_{1} K_{1}^{n}[s] .
$$


Obviously $V_{D_{1}}$ is a $K[s]$-module and therefore also a vector space over $K$. The counterpart of (1.5) is a realization

$$
G=W_{2}+P_{2} D_{2}^{-1} Q_{2}
$$

where $P_{2}$ and $Q_{2}$ are proper rational matrices, $W_{2}$ is strictly proper rational and $D_{2}$ is a polynomial matrix, $D_{2} \in K^{n_{2} \times n_{2}}$. Define

$$
U^{D_{2}}=K_{\infty}^{n_{2}}(s) /\left(K_{\infty}^{n_{2}}(s) \cap D_{2} s^{-1} K_{\infty}^{n_{2}}(s)\right) .
$$

Then $U^{D_{2}}$ is a $K_{\infty}(s)$-module and at the same time a $K$-vector space. At the end of this section we shall indicate why $U^{D_{2}}$ can be taken as a state space of a realization (1.2) of $\pi_{+} G$. Let us mention that the finite and infinite pole modules (see [9]) of $G(s)$ are given by $V_{D_{1}}$ and $U^{D_{2}}$, if (1.5) is an irreducible realization and (1.6) satisfies coprimeness conditions of the form (3.14).

We note that a nonsingular polynomial matrix $L \in K^{n \times n}[s]$ gives rise to two types of modules, namely the $K[s]$-module

$$
V_{L}=K^{n}[s] / L K^{n}[s]
$$

and the $K_{\infty}(s)$-module

$$
U^{L}=K_{\infty}^{n}(s) /\left(K_{\infty}^{n}(s) \cap L s^{-1} K_{\infty}^{n}(s)\right) .
$$

Beside realizations there is a wide range of issues such as similarity of state space models, system equivalence or simulation of restricted input output maps which involve two polynomial matrices $L$ and $L_{1}$ and homomorphisms from $V_{L}$ to $V_{L_{1}}$ and from $U^{L}$ to $U^{L_{1}}$. The $K[s]$-module homomorphisms from $V_{L}$ to $V_{L_{1}}$ are well understood. According to Fuhrmann [4 their description is based on intertwining relations between $L$ and $L_{1}$. In this note we study $K_{\infty}(s)$-module homomorphisms from $U^{L}$ to $U^{L_{1}}$. Our characterizations will be in correspondance with Fuhrmann's results in Ref. [2, 4]. Comparing the definitions of $V_{L}$ and $U^{L}$ we observe that $L K^{n}[s]$ is a submodule of $K^{n}[s]$ whereas in general $L s^{-1} K_{\infty}^{n}(s)$ is not contained in $K_{\infty}^{n}(s)$. Hence it is not surprising that $U^{L}$ is less easy to handle than $V_{L}$ and that in our study technical obstacles have to be removed which do not appear in the case of the module $V_{L}$.

To obtain a concrete representation of $U^{L}$ we define a map

$$
\rho^{L}: K_{\infty}^{n}(s) \rightarrow K^{n}[s]
$$

by

$$
\rho^{L} x=L \pi_{+} L^{-1} x, x \in K_{\infty}^{n}(s) .
$$


Put $\bar{x}=\rho^{L} x$. For $q \in K_{\infty}(s)$ and $\bar{x} \in \operatorname{Im} \rho^{L}$ we set $q \cdot \bar{x}=\overline{q x}$. This product is well defined since

$$
\operatorname{Ker} \rho^{L}=\left(K_{\infty}^{n}(s) \cap s^{-1} L K_{\infty}^{n}(s)\right) .
$$

Therefore $\operatorname{Im} \rho^{L}$ is a $K_{\infty}(s)$-module, isomorphic to the quotient module $U^{L}$ in (1.8). From now on we identify both modules such that

$$
U^{L}=\operatorname{Im} \rho^{L}=L \pi_{+} L^{-1} K_{\infty}^{n}(s) .
$$

Clearly, $U^{L}=0$ if $s L^{-1}$ is proper rational. A shift operator $S_{-}(L)$ on $U^{L}$ is given by

$$
S_{-}(L) \bar{x}=s^{-1} \cdot \bar{x}, \bar{x} \in U^{L} .
$$

Clearly, $S_{-}(L)$ is a nilpotent endomorphism of $U^{L}$.

Let us now give a concrete example for the use of $K_{\infty}(s)$-module $U^{L}$.Based on the representation (1.6) of $G$ we derive a realization of $\pi_{+} G$ having $U^{D_{2}}$ as its state space. We adapt a construction of [3]. Assume $\pi_{+} G(s)=$ $\sum_{\nu=0}^{t} G_{\nu} s^{\nu}$. Define the map $B_{2}: K^{p} \rightarrow U^{D_{2}}$ by

$$
B_{2} \xi=\rho^{D_{2}} Q_{2} \xi, \xi \in K^{p} .
$$

Put $N_{2}=S_{-}\left(D_{2}\right)$ and define $C_{2}: U^{D_{2}} \rightarrow K^{m}$ by

$$
C_{2} \bar{x}=-\left(P_{2} D_{2}^{-1} \bar{x}\right)_{0}, \bar{x} \in U^{D_{2}} .
$$

Then a straightforward computation yields

$$
G_{\nu}=-C_{2} N_{2}^{\nu} B_{2}, \nu=0,1, \ldots, t
$$

such that

$$
\sum_{\nu=0}^{t} G_{\nu} s^{\nu}=C_{2}\left(s N_{2}-I\right)^{-1} B_{2} .
$$

\section{Basic facts of the module $U^{L}$}

For a nonzero proper rational function $f=p / q, p, q \in K[s]$, let a degree function be defined by $\delta(p / q)=\operatorname{deg} q-\operatorname{deg} p$. It is well known that $\left(K_{\infty}(s), \delta\right)$ is a euclidean domain. The units $K_{\infty}^{*}(s)$ are the proper rational functions $f$ with $\delta f=0$. The ideal $\left(s^{-1}\right)$ is the unique maximal ideal of $K_{\infty}(s)$. Let us call a matrix $P \in K_{\infty}^{n \times n}(s)$ bicausal if $\operatorname{det} P \in K_{\infty}^{*}(s)$, i.e. if $P$ is invertible in $K_{\infty}^{n \times n}(s)$. If $W \in K^{m \times r}(s)$ has rank $n$ then there exist bicausal matrices $P$ and $Q$ such that

$$
W=P\left(\begin{array}{cc}
\Sigma & 0 \\
0 & 0
\end{array}\right) Q
$$


with

$$
\begin{aligned}
\Sigma=\operatorname{diag}\left(s^{-\alpha_{1}}, \ldots, s^{-\alpha_{t}}, s^{\beta_{t+1}}\right. & \left., \ldots, s^{\beta_{n}}\right) \\
& -\alpha_{1} \leq \cdots \leq-\alpha_{t}<0 \leq \beta_{t+1} \leq \cdots \beta_{n} .
\end{aligned}
$$

The integers $-\alpha_{1}, \ldots, \beta_{n}$ are uniquely determined by $W$. In particular, if $L \in K^{n \times n}[s]$ is nonsingular then

$$
s^{-1} L=P \Sigma Q
$$

for some $P, Q \in K_{\infty}^{n \times n}(s)^{*}$ and $\Sigma$ as in (2.1). In the case of a linear pencil $L(s)=A_{0}-A_{1} s$ the polynomials $s^{\alpha_{1}}, \ldots, s^{\alpha_{t}}$ are the elementary divisors of $A_{0} s-A_{1}$ belonging to the characteristic root 0 . According to [7] the matrix $\Sigma$ in (2.2) and (2.1) provides information on the structure of $U^{L}$. We have

$$
U^{L} \cong \oplus\left\{K_{\infty}(s) / s^{-\alpha_{j}} K_{\infty}(s), j=1, \ldots, t\right\}
$$

such that $U^{L}$ is a finitely generated torsion module over $K_{\infty}(s)$ with elementary divisors

$$
s^{-\alpha_{1}}, \ldots, s^{-\alpha_{t}} .
$$

We call (2.3) the infinite elementary divisors of $L$. Then $s^{\alpha_{1}}, \ldots, s^{\alpha_{t}}$ are the elementary divisors of the shift $S_{-}(L)$, and $\operatorname{dim}_{K} U^{L}=\alpha_{1}+\cdots+\alpha_{t}$. To describe a dual pairing [8] between the $K$-linear spaces $U^{L^{T}}$ and $U^{L}$ we note that

$$
\langle\bar{y}, \bar{x}\rangle=\left(y^{T} L^{-1} x\right)_{0}, \bar{y} \in U^{L^{T}}, \bar{x} \in U^{L},
$$

is a well defined nondegenerate bilinear form on $U^{L^{T}} \times U^{L}$.

\section{Homomorphisms}

Our main result is Theorem 3.3 below. Its proof will be based on the subsequent two lemmas. In the following $L \in K_{\infty}^{n \times n}(s)$ and $L_{1} \in K_{\infty}^{n_{1} \times n_{1}}(s)$ will be fixed nonsingular polynomial matrices.

Lemma 3.1. A map

$$
\Phi: K_{\infty}^{n}(s) \rightarrow U^{L_{1}}
$$

is a $K_{\infty}(s)$-module homomorphism if and only if there exists a matrix $\Theta \in$ $K_{\infty}^{n_{1} \times n}(s)$ such that

$$
\Phi x=\rho^{L_{1}}(\Theta x), x \in K_{\infty}^{n}(s) .
$$


Proof. Let $e_{1}, \ldots, e_{n}$ be the standard basis of $K^{n}$. Assume that $\Phi$ in (3.1) is a $K_{\infty}(s)$-module homomorphism. Then $\Phi e_{i}=\rho^{L_{1}} \theta_{i}$ for some $\theta_{i} \in K_{\infty}^{n_{1}}(s)$ and (3.2) holds with $\Theta=\left(\theta_{1}, \ldots \theta_{n}\right)$. The converse is obvious.

Condition (3.3) below together with a somewhat technical equivalent condition will be crucial.

Lemma 3.2. We have

$$
\Theta \operatorname{Ker} \rho^{L} \subseteq \operatorname{Ker} \rho^{L_{1}} .
$$

with $\Theta \in K_{\infty}^{n_{1} \times n}(s)$ if and only if there exist a matrix $\Theta_{1} \in K_{\infty}^{n_{1} \times n}(s)$ and a matrix $\Psi$ satisfying

$$
\Psi \in s^{-1} K_{\infty}^{n_{1} \times n}(s) \text { and } L_{1} \Psi \in K_{\infty}^{n_{1} \times n}(s)
$$

such that

$$
\left(\Theta+L_{1} \Psi\right) L=L_{1} \Theta_{1} .
$$

Proof. It is evident that (3.5) implies (3.3). To prove the converse implication we note that (3.3) is equivalent to $\Theta \operatorname{Ker} \rho^{L} \subseteq s^{-1} L_{1} K_{\infty}^{n_{1}}(s)$. If $s^{-1} L$ is factorized as in (2.2),

$$
\begin{aligned}
s^{-1} L=P \Sigma Q, \Sigma & =\operatorname{diag}(A, B), \\
& A=\operatorname{diag}\left(s^{-\alpha_{1}}, \ldots, s^{-\alpha_{t}}\right), B=\operatorname{diag}\left(s^{\beta_{t+1}}, \ldots, s^{\beta_{n}}\right)
\end{aligned}
$$

then $\operatorname{Ker} \rho^{L}=P \operatorname{diag}(A, I) K_{\infty}^{n}(s)$. Hence if

$$
G=L_{1}^{-1} \Theta P \operatorname{diag}(A, I)
$$

then (3.3) is equivalent to $G \in s^{-1} K_{\infty}^{n_{1} \times n}(s)$. From (3.6) and

$$
\Sigma=\operatorname{diag}(A, 0)+\operatorname{diag}(0, B)
$$

we obtain

$$
L_{1}^{-1} \Theta L=G \operatorname{diag}(I, 0) Q+L_{1}^{-1} \Theta P \operatorname{diag}(O, I) P^{-1} L .
$$

Now choose

$$
\Psi=-G \operatorname{diag}(I, O) Q .
$$

Then $\Psi$ satisfies (3.4) and if we put $\Theta_{1}=L_{1}^{-1} \Theta L+\Psi L$ then we have $\Theta_{1} \in K_{\infty}^{n_{1} \times n}(s)$, which proves (3.5).

We extend the map $\rho^{L_{1}}$ to $K^{n}(s)$ and define

$$
\rho_{e}^{L_{1}}=L_{1} \pi_{+} L_{1}^{-1} w, w \in K^{n}(s) .
$$


Theorem 3.3. The map $\phi: U^{L} \rightarrow U^{L_{1}}$ is a $K_{\infty}(s)$-module homomorphism if and only if there exist matrices $\Theta, \Theta_{1} \in K_{\infty}^{n_{1} \times n}(s)$ such that

$$
\Theta L=L_{1} \Theta_{1}
$$

and

$$
\phi \bar{x}=\rho_{e}^{L_{1}} \Theta \bar{x}, \bar{x} \in U^{L} .
$$

If (3.7) holds then we have

$$
\rho_{e}^{L_{1}} \Theta \bar{x}=\rho^{L_{1}} \Theta x
$$

for all $x \in K_{\infty}^{n}(s)$.

Proof. Let us show first that (3.7) implies (3.9). We have

$$
\begin{aligned}
& \rho_{e}^{L_{1}} \Theta \bar{x}=L_{1} \pi_{+} L_{1}^{-1} \Theta \bar{x}=L_{1} \pi_{+} \Theta_{1} L^{-1} \bar{x}= \\
& L_{1} \pi_{+} \Theta_{1} L^{-1} x=L_{1} \pi_{+} L_{1}^{-1} \Theta x=\rho^{L_{1}} \Theta x .
\end{aligned}
$$

Now let $\phi: U^{L} \rightarrow U^{L_{1}}$ be a $K_{\infty}(s)$-module homomorphism. Define $\Phi=\phi \rho^{L}$ such that

$$
\Phi x=\phi \bar{x}, x \in K_{\infty}^{n}(s) .
$$

Then $\Phi: K_{\infty}^{n}(s) \rightarrow U^{L_{1}}$ is also a $K_{\infty}(s)$-module homomorphism. Because due to Lemma 3.1 there exists a $\tilde{\Theta} \in K_{\infty}^{n_{1} \times n}(s)$ such that

$$
\Phi x=\rho^{L_{1}} \tilde{\Theta} x .
$$

It follows from (3.11) that $x, v \in K_{\infty}^{n}(s)$ and $\bar{x}=\bar{v}$ imply $\rho^{L_{1}} \tilde{\Theta} x=\rho^{L_{1}} \tilde{\Theta} v$. Therefore we obtain

$$
\tilde{\Theta} \operatorname{Ker} \rho^{L} \subseteq \operatorname{Ker} \rho^{L_{1}} .
$$

We can replace $\tilde{\Theta}$ in (3.12) and (3.13) by $\Theta=\tilde{\Theta}+L_{1} \Psi$ if $\Psi \in s^{-1} K_{\infty}^{n_{1} \times n}(s)$ and $L_{1} \Psi \in K_{\infty}^{n_{1} \times n}(s)$. From Lemma 3.2 we know that starting from (3.13) we can find a $\Psi$ which yields (3.7) with $\Theta_{1} \in K_{\infty}^{n_{1} \times n}(s)$. Thus we have shown that

$$
\phi \bar{x}=\rho^{L_{1}} \Theta x=\rho_{e}^{L_{1}} \Theta \bar{x}
$$

with $\Theta$ satisfying a relation (3.7).

Conversely, if a map $\phi: U^{L} \rightarrow U^{L_{1}}$ is defined by (3.7) and (3.8) then it is easy to verify that $\phi$ is a $K_{\infty}(s)$-module homomorphism. by

We remark that Theorem 3.3 remains true if condition (3.7) is replaced

$$
\pi_{+} L_{1}^{-1} \Theta=\pi_{+} \Theta_{1} L^{-1}
$$

Given the duality (2.4) between $U^{L}$ and $U^{L^{T}}$ it is not difficult to obtain the dual map of $\phi$. We set $\overline{\bar{w}}=\rho^{L_{1}^{T}} w, w \in K_{\infty}^{n_{1}}(s)$. 
Theorem 3.4. Let $\Theta, \Theta_{1} \in K_{\infty}^{n_{1} \times n}(s)$ be such that $\Theta L=L_{1} \Theta_{1}$. Let $\phi: U^{L} \rightarrow U^{L_{1}}$ be defined by (3.8). Then the dual map

$$
\phi^{*}: U^{L_{1}^{T}} \rightarrow U^{L^{T}}
$$

is given by

$$
\phi^{*} \overline{\bar{w}}=\rho^{L^{T}} \Theta_{1}^{T} w, \quad \overline{\bar{w}} \in U^{L_{1}^{T}} .
$$

We now turn to surjectivity and injectivity. For a pair $\Theta \in K_{\infty}^{n_{1} \times n}(s)$ and $L_{1} \in K^{n_{1} \times n_{1}}$ we set $\left(\Theta, s^{-1} L_{1}\right)_{l}=I$ if there exist proper rational matrices $C$ and $D$ such that

$$
\Theta C+s^{-1} L_{1} D=I .
$$

Similarly, for $\Theta_{1} \in K_{\infty}^{n_{1} \times n}(s)$ and $L \in K^{n \times n}$ we write $\left(\Theta_{1}, s^{-1} L\right)_{r}=I$ if $\left(\Theta_{1}^{T}, s^{-1} L^{T}\right)_{l}=I$.

Theorem 3.5. Let $\phi: U^{L} \rightarrow U^{L_{1}}$ be defined by (3.9) and (3.7). Then

(i) $\phi$ is surjective if and only if $\left(\Theta, s^{-1} L_{1}\right)_{l}=I$,

(ii) $\phi$ is injective if and only if $\left(\Theta_{1}, s^{-1} L\right)_{r}=I$.

Proof. (i) Assume first that $\phi$ is surjective. Let $w \in K_{\infty}^{n_{1}}(s)$ be given. Then $\rho^{L_{1}} w=\rho^{L_{1}} \Theta v$ for some $v \in K_{\infty}^{n}(s)$. We have $w-\Theta v \in \operatorname{Ker} \rho^{L_{1}}$, which implies

$$
w \in \Theta K_{\infty}^{n}(s)+s^{-1} L_{1} K_{\infty}^{n}(s)
$$

or equivalently $\left(\Theta, s^{-1} L_{1}\right)_{l}=I$. Conversely, suppose that (3.14) holds. To show that $w=\rho^{L_{1}} x$ is in $\phi U^{L}$ we note that (3.14) implies $x=\Theta v+s^{-1} L_{1} x_{2}$ for some $v \in K_{\infty}^{n}(s), x_{2} \in K_{\infty}^{n_{1}}(s)$. Because of $s^{-1} L_{1} x_{2} \in \operatorname{Ker} \rho^{L_{1}}$ we obtain $w=\rho^{L_{1}} \Theta v=\phi \bar{v}$.

(ii) By duality the statement follows at once from (i).

If $M$ is a finitely generated $p$-module over a principal ideal domain and $S$ is a submodule and $Q$ is a quotient module of $M$ then the relations between the invariants of $M$ and those of $S$ and $Q$ are well known (see e.g. [5, p. $92,93])$. We complete our note with a corresponding observation on the existence of surjective and injective homomorphisms. Let

$$
s^{-\alpha_{1}}, \ldots, s^{-\alpha_{t}}, \alpha_{1} \geq \cdots \geq \alpha_{t}
$$

and

$$
s^{-\gamma_{1}}, \ldots, s^{-\gamma_{p}}, \gamma_{1} \geq \cdots \geq \gamma_{p}
$$

be the infinite elementary divisors of $L$ and $L_{1}$, respectively. Then there exists a surjective $K_{\infty}(s)$-module homomorphism $\phi: U^{L} \rightarrow U^{L_{1}}$ if and only if

$$
t \geq p \quad \text { and } \quad \alpha_{1} \geq \gamma_{1}, \ldots, \alpha_{p} \geq \gamma_{p}
$$


and there exists an injective $\phi$ if and only if

$$
t \leq p \quad \text { and } \quad \alpha_{1} \leq \gamma_{1}, \ldots, \alpha_{t} \leq \gamma_{t}
$$

\section{References}

[1] G. Conte and A.M. Perdon, Generalized state-space realizations for nonproper rational transfer functions, Systems Control Lett. 1(1981), 270276.

[2] P.A. Fuhrmann, Algebraic system theory: an analyst's point of view, J. Franklin Inst. 301(1976), 521-540.

[3] P.A. Fuhrmann, On strict system equivalence, Internat J. Control 25 (1977), 5-10.

[4] P.A. Fuhrmann, Linear Systems and Operators in Hilbert Space, McGraw-Hill, New York, 1981.

[5] P. Ribenboim, Rings and Modules, Interscience, New York, 1969.

[6] H.H. Rosenbrock, Structural properties of linear dynamical systems, Internat. J. Control 20(1974), 191-202.

[7] H.K. Wimmer, The structure of nonsingular polynomial matrices, Math. Systems Theory 14(1981), 367-379.

[8] H.K. Wimmer, Polynomial matrices and dualities, Systems Control Lett. 1(1981), 200-203.

[9] B.F. Wyman, M.K. Sain, G. Conte and A.M. Perdon, Poles and zeros of matrices of rational functions, Linear Algebra Appl. 157(1991), 113-139. 